\title{
COMPUTER SIMULATIONS OF ASTRONOMICAL PHENOMENA
}

\author{
A. Noels and R. Papy \\ Institut d'Astrophysique, Université de Liège, B-4200 Ougrée-Liège, Belgium
}

\section{Introduction}

The basic idea of this project is to introduce a senior student to an astronomical phenomenon that he analyses carefully and simulates on the graphic screen of a personal computer. In a second step, this simulation serves as a demonstration for junior students.

Three examples are presented here: the retrograde motion of the planets, the Earth's rotation, and the precession and the equilibrium figure of the Earth due to tides. Programs have been written on an IBM PC/AT.

It would be highly desirable to start an exchange program of material of didatic interest among the international astronomical community ${ }^{1}$

\section{The Retrograde Motion of the Planets}

In a first option, a part of the celestial sphere centered on the ecliptic circle is developed along a cylinder; the ecliptic latitudes are magnified. As many as five planets can be visualized simultaneously with different colors. Observations can be made for any planet chosen in the menu. For a terrestrial observer, the annual motion of the sun is a horizontal mid-screen track. For a given planet, the time interval between two successive retrograde loops is its synodic period. Observed from the Earth, the exterior planets present a loop nearly every year while the interior planets show a loop nearly each time they make a revrlution around the sun.

The second option shows the chosen planets and the Earth orbiting the sun, as seen from the ecliptic pole, while their apparent paths are represented in the lower part of the screen as in option 1 but with a smaller magnification in latitude.

A third option gives the apparent orbits of the planets in the ecliptic plane, viewed from a distant point on an axis perpendicular to the ecliptic plane. The sun orbits the Earth and the planets show their retrograde loops in the ecliptic plane. The origin of the deferents and epicycles in the Ptolemaic system is clear. The lower portion of the screen still shows the same tracks as in option 1.

\footnotetext{
${ }^{1}$ Anyone interested should contact A. Noels, Institut d'Astrophysique, 5 Avenue de Cointe, B-4200 Ougrée-Liège, Belgium.
} 


\section{The Earth's Rotation - Precession}

The rotation of the Earth is viewed in an inertial frame centered on the Earth. In that frame, stars would occupy fixed points on the screen and the horizontal $(x, y)$ plane is the ecliptic plane. A red meridian serves as a reference, and a red spot is the place on Earth where the mean sun is at the zenith at local noon time. A red $\gamma$ marks the vernal equinox. The origin of time (year 0 , day 0 , hour 12) is arbitrarily taken when the reference meridian, $\gamma$, and the mean sun coincide.

In the first mode, the time interval is 12 solar minutes and the diurnal rotation of the Earth is seen. A solar day is equivalent to $24 \mathrm{~h}$. Starting from the mean sun, marked by the red spot, the red meridian makes more than a complete revolution in $24 \mathrm{~h}$, to coincide again exactly with the mean sun; $\gamma$ remains fixed on the screen. The reference meridian makes a complete revolution in $24 \mathrm{~h}$.

The second mode has a time interval of 2 solar days. It shows the annual revolution of the Earth around the sun. The angular distance between the reference meridian and the mean sun is constant and $\gamma$ is nearly fixed on the screen. The red spot makes a whole revolution in a tropical year, as does the reference meridian, which demonstrates the difference between the sidereal and the solar day.

The time interval is set to 72 years in the third mode to show the precession of the axis of rotation. The $\gamma$ point makes a complete revolution in the retrograde sense in about 26000 years.

\section{The Equilibrium Figure of the Earth due to Tides}

The tidal effect of the sun and the moon is simulated, assuming the Earth to be a homogeneous spheroid. The Earth's center occupies the center of the screen and the positions of the moon and the sun are computed with great accuracy as a function of time. The distance ratio is not to scale and the deformation of the Earth is magnified by a constant factor. The sun is at a fixed point at the right of the screen while the moon moves around the Earth. The equilibrium figures of the Earth due to the tidal force of a single body are drafted in white and the resulting figure is fully painted. Near the sun, a red line scales the Earth-moon distance and a white line scales the flattening of the Earth due to the presence of the moon. The equilibrium figure of the Earth is the same whenever the Earth, the moon, and the sun are on the same line, whether in opposition or in conjunction, the only possible difference coming from a change in the Earth-moon distance. 